\title{
Terminal Doppler Weather Radar (TDWR) Observation of Atmospheric Flow over Complex Terrain during Tropical Cyclone Passages
}

\author{
C.M. Shun ${ }^{*}$ and S.Y. Lau \\ Hong Kong Observatory, 134A Nathan Road, Kowloon, Hong Kong
}

\begin{abstract}
To facilitate warning of low-level wind shear associated with convective storms, a Terminal Doppler Weather Radar (TDWR) was installed about $12 \mathrm{~km}$ to the northeast of the Hong Kong International Airport (HKIA). The HKIA is located just off the northern shore of an island known as Lantau. The HKIA lies on the lee side of the complex terrain of Lantau when winds come from the east through southwest.

With the commissioning of the TDWR in 1997, interesting high-resolution radar data were collected in strong southerly flows during tropical cyclone passages. These data sets reveal the complex low-level atmospheric flow in the vicinity of the HKIA, including streaks of low-speed flow, reverse flows, small-scale vortices and high-speed gap flows. Animation sequences of the radar images suggest existence of von Kármán vortex streets and vortex shedding in the wake regions. These phenomena could induce strong shear regions which led to significant low-level wind shear for landing/departing aircraft. Analysis of on-board flight data for a wind shear event experienced by a landing aircraft in strong southeasterly flow revealed that terrain-induced features with horizontal scale less than $1 \mathrm{~km}$ brought significant air speed changes to the aircraft over a short duration of time.
\end{abstract}

Keywords: Terrain-induced wake, low-level wind shear, vortex shedding, Doppler weather radar

\section{INTRODUCTION}

The new Hong Kong International Airport (HKIA) at Chek Lap Kok (CLK) came into operation on 6 July 1998. The airport has two parallel northeast-southwest oriented runways designated as RWY 07L/25R and RWY 07R/25L. As part of a comprehensive set of meteorological systems implemented to support the HKIA, the Hong Kong Observatory installed a Terminal Doppler Weather Radar (TDWR) for detecting microburst and wind shear associated with convective storms ${ }^{1,2}$.

Lantau Island, immediately south of the HKIA, is quite mountainous. Figure 1 illustrates the complex terrain of Lantau Island and location of the HKIA. The northeast-southwest oriented island has a width of about $5 \mathrm{~km}$ and length of about 20 km. In the middle of Lantau, Nei Lak Shan (751 m), Lantau Peak (934 m), Sunset Peak (869 m) and Lin Fa Shan (766 m) form a U-shape ridge. These peaks rise to between 750 and $950 \mathrm{~m}$ above mean sea level (amsl) with valleys as low as 350 to $450 \mathrm{~m}$ amsl separating these peaks. To the southwest of this U-shape ridge, a northeast-southwest oriented ridge rises to between 400 and $500 \mathrm{~m}$ amsl at many locations along the ridge line. Locations of ground based anemometers installed over and in the vicinity of the HKIA are also indicated in Figure 1 to facilitate the discussions below.

Study in 1994 using research aircraft suggested the occurrence of terrain-induced turbulence in association with airflow over Lantau. ${ }^{3}$. Two distinct mechanisms, gravity wave - critical level flow interaction and mechanically generated turbulence under deep uniform flow, were attributed as the main causes of such terrain-induced turbulence. However, pilot reports received since opening of the HKIA indicated that, apart from terrain-induced turbulence, significant terrain-induced wind shear could also occur downwind of Lantau in both stably stratified conditions and deep uniform flow. An account of the former phenomenon was recently presented in the $9^{\text {th }}$ Conference on Mountain Meteorology ${ }^{4}$.

* Correspondence: Email: cmshun@hko.gcn.gov.hk; Telephone: (852) 2926 8435; Fax: (852) 23119448 
This paper gives an account of the complex atmospheric flow and terrain-induced wind shear phenomena observed by the TDWR during several cases of tropical cyclone passages in 1997 and 1999. Their effects on aircraft operations will also be discussed.

\section{TERMINAL DOPPLER WEATHER RADAR IN HONG KONG}

The TDWR is strategically located at Tai Lam Chung (Figure 1), about $12 \mathrm{~km}$ northeast of the HKIA so that it has a clear view of the runways, airport approach and departure areas. Its direct line-of-sight towards the airport is almost aligned with the orientation of the runways so that the Doppler radial velocity observed provides a good estimate of the head/tail wind and hence also wind shear experienced by landing/departing aircraft. To avoid beam blockage by nearby ships, the antenna of the TDWR was installed at about $60 \mathrm{~m}$ amsl. The TDWR is essentially the same system installed by the US Federal Aviation Administration (FAA) ${ }^{5,6}$ at more than 40 airports in the USA, with a number of local enhancements in Hong Kong of communications and computers, and customization of displays and data presentation formats. The radar is a $250 \mathrm{~kW}$ peak power C-band system with a high performance antenna with half-power beamwidth of 0.55 degree. It operates a highly stable klystron based amplifier which allows clutter suppression of up to $55 \mathrm{~dB}$. Although the TDWR was designed to detect microburst and wind shear associated with convective storms, it is highly sensitive and can provide high-resolution data under both rainy conditions and favourable clear air conditions.

The TDWR routinely makes Plan Position Indicator (PPI) scans at different elevation angles depending on the scan strategy. Base data including reflectivity, Doppler radial velocity, spectrum width and signal-to-noise ratio with resolution of $150 \mathrm{~m}$ in range and 1 degree in azimuth are provided for each scan. The lowest elevation angle employed for automatic wind shear detection is 0.6 degree. Higher elevation angles at 1.0 (and 1.1), 2.4, 6.0, .., up to 60.0 degrees are also used by the microburst and gust front detection algorithms. When either wind shear or significant precipitation area is detected by the TDWR, quick sector scans will be made over the airport approach and departure areas so that the lowest elevation angle at 0.6 degree can be repeated in less than one minute intervals for automatic wind shear detection.

Since the TDWR has to operate in a high clutter environment, sophisticated clutter removal algorithms are used to provide high quality base data for automatic wind shear detection. These algorithms include the use of (i) $55 \mathrm{~dB}$ frequency domain filters which provide high-pass band filtering of targets with small or no Doppler component; (ii) Clutter Residue Editing Maps (CREMs) for removal of residual stationary clutters at 0.6, 1.0 (and 1.1), 2.4 and 6.0 degrees; (iii) clutter polygons to eliminate multi-path or sidelobe contamination at 0.6 and 1.0 degree for the area south of the Lantau Island; and (iv) point target filters which smooth spike-like anomalies in radar reflectivity owing to moving point clutters like aircraft, birds, automobiles and marine vessels.

The system also implements velocity dealiasing, signal-to-noise thresholding and range obscuration editing algorithms to ensure that the Doppler radial velocity data of the lowest elevation angle scan at 0.6 degree is free from contamination by aliased velocities, low signal-to-noise data and range folding. In particular, two consecutive scans are routinely made at 0.6 degree with different Pulse Repetition Frequecies (PRFs) at $598 \mathrm{~Hz}$ and $838 \mathrm{~Hz}$ for velocity dealiasing. The dealiased data is then used to form a portion of a windfield model for use in dealiasing of subsequent scans at higher elevation angles which do not employ dual scans in order to save time for more frequent updates of data.

After processing of the radar base data to remove data contamination and radar ambiguities, the conditioned data are processed by several weather detection and warning algorithms for automatic generation of alphanumeric, alarm and graphical products for the end users. The radar data presented in this paper are all conditioned data.

\section{FIRST OBSERVATION OF VELOCITY STREAKS AND REVERSE FLOWS}

Typhoon Victor was the first tropical cyclone which landed over Hong Kong since the TDWR was put into trial operation in early 1997. Victor formed to the south of Hong Kong on 31 July 1997 and intensified into a typhoon with estimated maximum winds of about $33 \mathrm{~m} \mathrm{~s}^{-1}(64 \mathrm{kt})$ on 2 August 1997. It moved north and landed over Hong Kong at about 12 UTC on the same day. It also weakened to a severe tropical storm with estimated maximum winds of about $31 \mathrm{~m} \mathrm{~s}^{-1}(60 \mathrm{kt})$ at the 
same time. Its centre just skirted the northeastern tip of Lantau Island and came within $8 \mathrm{~km}$ east of the TDWR. Gale force northwesterly winds were recorded over CLK. As Victor moved further inland, the winds at CLK gradually turned southwesterly and the TDWR wind shear detection algorithms started to produce wind shear alerts of $25 \mathrm{kt}\left(13 \mathrm{~m} \mathrm{~s}{ }^{-1}\right) \mathrm{speed}$ loss (i.e. a warning of a loss of air speed of $25 \mathrm{kt}\left(13 \mathrm{~m} \mathrm{~s}^{-1}\right)$ to an aircraft due to a local divergent airflow) over the eastern approach/departure area at 13:20 UTC. Figure 2 shows the TDWR radial velocity data at 0.6-degree elevation at 14:02 UTC when $60 \mathrm{kt}\left(\sim 30 \mathrm{~m} \mathrm{~s}^{-1}\right)$ speed loss was detected over the eastern approach/departure area of the southern runway. In the figure, the detected wind shear locations are indicated in the form of red "bandaid" shapes with speed losses given in knots at the centre of these shapes.

Analysis of Figure 2 reveals maximum radial winds of up to about $-30 \mathrm{~m} \mathrm{~s}^{-1}$ (i.e., towards the radar or inbound) over and to the east of CLK. These agree well with the maximum 1-minute southwesterly wind gust of about $28 \mathrm{~m} \mathrm{~s}^{-1}$ recorded by the surface anemometer at R1E (see Figure 1 for location of R1E). Greatly contrasting this were very small negative radial winds observed near the coast of Lantau to the south of CLK. Without going into details of the TDWR wind shear detection algorithms, the detected wind shear of $60 \mathrm{kt}\left(\sim 30 \mathrm{~m} \mathrm{~s}^{-1}\right)$ speed loss can apparently be explained by this significant difference of radial winds over the shear region. This significant shear condition actually persisted during the next few hours until 18:46 UTC when the southwesterly winds gradually weakened as Victor moved further inland.

Over this strong shear region in Figure 2, it is not difficult to see streaks of smaller inbound radial velocity emanating from the terrain of Lantau Island downwind of the U-shape ridge. In an animation sequence of the 0.6-degree radial velocity imageries, these streaks displayed wavy characteristics and very often became unstable and broke up into several small pockets of local radial velocity minima. The width of these low (radial) speed streaks and the size of the low (radial) speed pockets were of the order of $1 \mathrm{~km}$ or less. The radial velocities of these streaks and pockets were typically $10 \mathrm{~m} \mathrm{~s}^{-1}$ less than that of the ambient flow.

Similar low-speed streaks and pockets can also be seen at 1.0 degree (Figure 3) and 2.4 degrees* (Figure 4) elevations. More interestingly, regions of positive (outbound) radial velocities can clearly be seen just downwind of the individual peaks (Lin Fa Shan, Lantau Peak and Nei Lak Shan) of the U-shape ridge in Figure 4, and to a much lesser extent, in Figure 3. Ignoring spikes of dubious data, the outbound radial velocities downwind of Lin Fa Shan, Lantau Peak and Nei Lak Shan in Figure 4 reached $8 \mathrm{~m} \mathrm{~s}^{-1}, 5 \mathrm{~m} \mathrm{~s}^{-1}$ and $3 \mathrm{~m} \mathrm{~s}^{-1}$ respectively. While the outbound radial velocities stayed rather close to the lee of Nei Lak Shan, the two other regions extended to about $1.5 \mathrm{~km}$ downwind of Lantau Peak and Lin Fa Shan. An animation sequence of the 2.4-degree radial velocity imageries revealed that these two regions occasionally shed smaller outbound velocity regions which propagated downstream in the prevailing flow direction. While propagating downstream, these smaller outbound velocity regions quickly lost their outbound velocities but remained as part of the low-speed streaks which emanated from the peaks. Sometimes their identities could be traced as individual low-speed pockets while propagating downstream, especially when they were still close to the peaks. However, they became rather difficult to trace as individual entities further downstream. In Figure 3, careful analysis also revealed tiny outbound velocity regions close to Nei Lak Shan and Lantau Peak. Although one might challenge that these tiny regions could be spikes of dubious data, their existence was supported by an animation sequence which revealed consistently good data quality and their persistence over the same regions as identified in the 2.4-degree elevation scan data.

\section{CONCEPTUAL MODEL FOR THE OBSERVED STREAKS AND REVERSE FLOWS}

Taking into account the above analyses of data at 0.6-, 1.0- and 2.4-degree elevations, it is not difficult to argue that the observed low-speed streaks and pockets were formed by vortex shedding downstream of the individual peaks of the Ushape ridge of Lantau Island. This is strongly supported by the reverse flows and occasional shedding of outbound velocity regions observed on the immediate lee slopes of these peaks at 2.4-degree elevation. Based on the locations of the highspeed flows on either side of Lin Fa Shan, Lantau Peak and Nei Lak Shan, the diameters of the recirculation vortices are estimated to be around $2 \mathrm{~km}, 1.5 \mathrm{~km}$ and slightly less than $1 \mathrm{~km}$ respectively. Furthermore, the reverse flows on the lee slopes of Lantau Peak and Nei Lak Shan observed at 2.4 degrees (i.e. at around $700 \mathrm{~m}$ amsl) were also marginally observed at 1.0 degree (i.e. at around $350 \mathrm{~m}$ amsl). This suggests that the recirculation vortices were probably formed above $350 \mathrm{~m}$

\footnotetext{
${ }^{*}$ Noting that clutter polygons are not installed for 2.4-degree elevation scans, care must be exercised to interpret Figure 4 and other 2.4degree elevation scan imageries south of the U-shape ridge due to possible data contamination by sidelobe returns from aloft.
} 
amsl, which is roughly the level of the valleys between Nei Lak Shan, Lantau Peak and Sunset Peak. These recirculation vortices are apparently sources of significant shear vorticity. This is evidenced by the lateral shear resulting from some 30 $\mathrm{m} \mathrm{s}^{-1}$ inbound high-speed flows and about 3-8 $\mathrm{m} \mathrm{s}^{-1}$ reverse flows, separated only by a horizontal distance of a couple of kilometers or less. Although the shed vortices quickly lost their outbound velocities as they propagated downstream, they remained in the form of low-speed streaks/pockets which still possessed shear vorticity of significant magnitude. Also the locations and orientations of the streaks observed at different elevation angles (and hence altitudes) were rather consistent, strongly suggesting that the streaks/pockets observed at lower elevation angles/altitudes were formed by vorticity transported downwards from the streaks/pockets generated aloft.

If it is accepted that vorticity was generated on the lee slopes above the valleys, advected downstream and transported to lower altitudes, a natural question would be whether vorticity was also transported to higher altitudes considering the nearneutral vertical profile. The average Brunt-Väisälä frequency was estimated to be $0.012 \mathrm{~s}^{-1}$ in the lowest $1500 \mathrm{~m}$ amsl from the radiosonde ascent at 18 UTC on 2 August 1997 launched at King's Park, around $25 \mathrm{~km}$ east of CLK. In the TDWR radial velocity data at 6.0-degree elevation at 14:07 UTC on 2 August 1997 (not shown), it was found that the southwesterly flow above the U-shape ridge was disturbed with regions of higher radial velocities above the ridges. A low-speed pocket could also be found to the northeast of Lin Fa Shan at an altitude of about $950 \mathrm{~m}$ amsl. There was no signature of the streaks observed at elevation angles lower than 6.0 degrees.

Very similar streaks could be identified downwind of the U-shape ridge when we reviewed the 6.0-degree elevation scan data collected during the passage of Typhoon York at 10:01 UTC on 16 September 1999 (Figure 5). At this time, York was about $50 \mathrm{~km}$ west of the TDWR after crossing the southwestern part of Lantau, bringing very strong southwesterly flow to the CLK area.

It is therefore apparent in the case of York that vorticity was also transported to higher altitudes. For example, the lowspeed pocket just to the northeast of Lin Fa Shan in Figure 5 was observed between 950 and $1050 \mathrm{~m}$ amsl, above Lantau Peak at $934 \mathrm{~m}$ amsl - the highest peak of Lantau. Very large shear vorticity was also associated with this pocket which had inbound radial velocity as low as $7 \mathrm{~m} \mathrm{~s}^{-1}$ in its core and high-speed inbound flow of up to about $30 \mathrm{~m} \mathrm{~s}^{-1}$ within $1 \mathrm{~km}$ west of the core.

\section{TERRAIN-INDUCED WIND SHEAR EXPERIENCED BY AIRCRAFT}

Since opening of the HKIA on 6 July 1998, with assistance of airlines and pilots, aircraft reports and on-board flight data were provided to us to help analyze wind shear events. The following discussion presents one of these events which occurred during the passage of Tropical Storm Maggie during the night of 7 June 1999.

In the early morning of 7 June 1999, Typhoon Maggie made landfall over the northeastern part of Hong Kong and moved southwest very quickly. After it departed Hong Kong, it slowed down and made a loop at about $250 \mathrm{~km}$ southwest of Hong Kong and moved northwards in the evening. The winds in Hong Kong strengthened again from the south to southeast as a result. At HKIA, winds with 1-minute mean speed reaching $18 \mathrm{~m} \mathrm{~s}^{-1}$ (35 kt) and 1-minute gusts reaching $27 \mathrm{~m} \mathrm{~s}^{-1}(52 \mathrm{kt})$ were recorded. Winds at YTS and NLS (see Figure 1 for locations of YTS and NLS) were even stronger, with 1-minute mean speed reaching $34 \mathrm{~m} \mathrm{~s}^{-1}(66 \mathrm{kt})$ at YTS and 1-minute gusts reaching $39 \mathrm{~m} \mathrm{~s}^{-1}(76 \mathrm{kt})$ at NLS. The King's Park radiosonde ascent at 12 UTC on 7 June 1999 indicated that the profile was essentially near-neutral with the average BruntVäisälä frequency estimated to be $0.012 \mathrm{~s}^{-1}$ in the lowest $1500 \mathrm{~m}$ amsl. During the night of 7 June 1999 and early morning of 8 June 1999, several aircraft reports of wind shear were received and six arrival flights went around on account of the wind shear and turbulence conditions. Two of these arrival flights finally diverted to neighbouring airports following abortive approaches.

Figure 6 shows the on-board flight data of altitude, ground speed (speed of aircraft relative to the ground) and air speed (speed of aircraft relative to the wind) of a flight which went around at 13:16 UTC while approaching the southern runway from the west (RWY 07R). It is obvious that the approach of this flight was already rather turbulent with the air speed fluctuated between $130 \mathrm{kt}\left(67 \mathrm{~m} \mathrm{~s}^{-1}\right)$ and $150 \mathrm{kt}\left(77 \mathrm{~m} \mathrm{~s}^{-1}\right)$ at high frequencies when the aircraft was descending from $1300 \mathrm{ft}$ $(400 \mathrm{~m})$ to $200 \mathrm{ft}(60 \mathrm{~m})$ amsl. The air speed also deviated from the ground speed significantly for a few short intervals, the first one being an air speed increase of $20 \mathrm{kt}\left(10 \mathrm{~m} \mathrm{~s}^{-1}\right)$ followed by a decrease of $16 \mathrm{kt}\left(8 \mathrm{~m} \mathrm{~s}^{-1}\right)$ when the aircraft was at about $1200 \mathrm{ft}(370 \mathrm{~m})$ amsl. Above all, the most significant wind shear that the aircraft experienced was between 13:16:33 


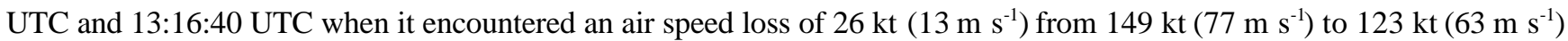
at about $200 \mathrm{ft}(60 \mathrm{~m})$ amsl. As a result of this air speed loss, the descent of the aircraft was much steeper than the normal 3 -degree profile. Based on the drop in altitude, the maximum instantaneous rate of descent was estimated to be around 2000 $\mathrm{ft} \min ^{-1}\left(10 \mathrm{~m} \mathrm{~s}^{-1}\right)$ at 13:16:41 UTC. The on-board wind shear alarm was triggered as a result and the pilot initiated a goaround. Within a few seconds after this, the air speed recovered partly due to increased thrust and ground speed and partly as a result of an environmental headwind gain of around $20 \mathrm{kt}\left(10 \mathrm{~m} \mathrm{~s}^{-1}\right)$.

Noting that the winds were from the southeast, the aircraft should be within the wake region of the northeast-southwest oriented ridge southwest of the U-shape ridge mentioned in Section 1 when it was below $1300 \mathrm{ft}$ (400 m). Knowing further that the radar reflectivity (not shown) was rather weak (less than $30 \mathrm{dBZ}$ ) over and in the vicinity of the approach area, it is likely that the wind shear was caused by terrain-induced features rather than convective downdraft.

Figure 7 shows the TDWR radial velocity data at 0.6-degree elevation at 13:16:03 UTC on 7 June 1999, 30 seconds before the air speed loss was encountered by the aircraft. The northeast-southwest oriented zero isodop in the figure clearly indicates that the low-level winds were from the southeast and therefore the winds had very small component in the radial direction relative to the radar beams over the airport western approach area. Nevertheless, the radial velocity data shows that the flow on the lee of the U-shape ridge was highly disrupted by terrain. Small areas of outbound radial velocities are found embedded in inbound radial velocities. In particular, an area of inbound radial velocities of up to $9 \mathrm{~m} \mathrm{~s}^{-1}$ can be identified over the touchdown zone of RWY 07R at an altitude of about $220 \mathrm{~m}(720 \mathrm{ft})$ amsl on the lee of Nei Lak Shan in Figure 7. The size of this area is less than $1 \mathrm{~km}$ across. To an aircraft approaching from the west, this area of inbound radial velocities alone would lead to an increase of tailwind and hence air speed loss of about $9 \mathrm{~m} \mathrm{~s}^{-1}$ or $18 \mathrm{kt}$. Furthermore, noting that there were also outbound radial velocities of up to $6 \mathrm{~m} \mathrm{~s}^{-1}$ very close to this area (see inset of Figure 7), it is not surprising that the aircraft experienced a total loss of $26 \mathrm{kt}\left(13 \mathrm{~m} \mathrm{~s}^{-1}\right)$ assuming that similar conditions also affected altitudes lower than that of the radar beam at 0.6-degree elevation. Figure 6 also indicates that the air speed did increase by $13 \mathrm{kt}(7$ $\left.\mathrm{m} \mathrm{s}^{-1}\right)$ from $136 \mathrm{kt}\left(70 \mathrm{~m} \mathrm{~s}^{-1}\right)$ to $149 \mathrm{kt}\left(77 \mathrm{~m} \mathrm{~s}^{-1}\right)$ in one second at 13:16:32 UTC. However, no large outbound radial velocity area could be found in Figure 7 to fully explain the subsequent air speed gain of around $20 \mathrm{kt}\left(10 \mathrm{~m} \mathrm{~s}^{-1}\right)$. This discrepancy is probably due to the small scale of the features concerned and the fact that the radar beam at 0.6-degree elevation was at an altitude of about $220 \mathrm{~m}$ or $720 \mathrm{ft}$ amsl over the touchdown zone of RWY 07R. Nevertheless, the whole wind shear event only spanned a total of 13 seconds which, for the aircraft travelling at a ground speed of 140-145 kt (72-75 $\mathrm{m} \mathrm{s}^{-1}$ ), corresponded to a distance of about $950 \mathrm{~m}$. This is consistent with the scale of the features identified in Figure 7.

\section{TERRAIN-INDUCED VORTICES AND HIGH-SPEED GAP FLOWS}

The wind shear event described in the last section revealed that small-scale terrain-induced features on the lee of Nei Lak Shan were responsible for the significant air speed changes over a short duration of time. These small-scale features might be vortices or eddies generated by the disruption of the strong southeasterly flow by the complex terrain of Lantau. The lack of radial velocity component relative to the radar beams however made it rather difficult to identify the detailed structure of these features and track their development and movement. Fortunately, the winds veered more towards the south during the night, allowing the TDWR to pick up interesting phenomena around 19 UTC on 7 June 1999.7 These phenomena provide some insight about the characteristics of the terrain-induced features.

Figure 8 shows the 0.6-degree elevation radial velocity data at 18:50 UTC on 7 June 1999. In additional to the numerous northeast-southwest oriented streaks of high- and low-speed inbound radial velocities, two small-scale vortices with dipoles of large inbound radial velocities and small outbound radial velocities can be identified. One was located to the southeast of the airport on the lee of Lin Fa Shan (vortex A), and the other one to the southwest of the airport on the lee of Cheung Shan (vortex B) (see Figure 1 for location of Cheung Shan). The magnitudes of the inbound (and outbound) radial velocities associated with A and B were respectively $-22(+4) \mathrm{m} \mathrm{s}^{-1}$ and $-16(+2) \mathrm{m} \mathrm{s}^{-1}$. While it was difficult to trace vortex B in the subsequent radar imageries, probably due to its quick dissipation coupled with inadequate signal-to-noise-ratio in the radar data, vortex A could be traced in each subsequent radar imagery until 18:55 UTC (see Figures 9 and 10). Although the small outbound radial velocity of the dipole could no longer be observed after 18:50 UTC, the area of large inbound radial velocity could be seen throughout with small inbound radial velocity to its immediate southeast. The magnitudes of the large (and small) inbound radial velocities associated with A at 18:53 UTC were $-21(-4) \mathrm{m} \mathrm{s}^{-1}$. Considering the size of only about $1 \mathrm{~km}$ across, very large shear vorticity was apparently maintained by A. 
Examination of data at 1.0-degree elevation (not shown) revealed that the size, strength and location of A resembled those observed at 0.6-degree. Although the identity of A at higher altitudes was subject to argument when data at 2.4-degree elevation was examined, the locations of the large and small inbound radial velocities were highly consistent at 0.6-, 1.0and 2.4-degree elevations. This strongly suggests that A had a vertically aligned structure with very large shear vorticity in the horizontal. Although reverse flows on the lee slopes of the various peaks coupled with the ambient flow aloft could suggest the existence of rotors in the conventional sense, i.e. with horizontal axis of rotation, there is no evidence to suggest that $\mathrm{A}$ was a rotor. Based on the locations of $\mathrm{A}$ in the 0.6-degree elevation imageries, its speed of movement (towards the northeast) was in the range of $15-20 \mathrm{~m} \mathrm{~s}^{-1}$, consistent with but slightly higher than the low-level wind speeds measured by the airport anemometers.

It is interesting to note that, compared with Figure 8, Figure 9 shows that streaks of high inbound radial velocities became more prominent at 18:53 UTC. Indeed, the TDWR wind shear detection algorithms also started to give out wind shear alerts of 20-40 kt $\left(\sim 10-20 \mathrm{~m} \mathrm{~s}^{-1}\right)$ at the same time. Examination of these alerts (not shown) revealed that they were associated with the high-speed streak over the airport (streak I) and another high-speed streak to the northeast of the Ushape ridge (streak II). The high-speed streaks became even more prominent at 18:55 UTC (Figure 10) with a third one (streak III) forming in the middle of these earlier two upstream of vortex A. The inbound radial velocities of these highspeed streaks were generally around $20 \mathrm{~m} \mathrm{~s}^{-1}$ with maximum reaching $24-26 \mathrm{~m} \mathrm{~s}^{-1}$. In contrast, the inbound radial velocities of the low-speed streaks between these high-speed streaks were just a few metres per second. Each of these high-speed streaks coupled with an adjacent low-speed streak to its west would apparently lead to large divergent shear.

Analysis of data at 1.0-degree (not shown) and 2.4-degree (Figure 11) elevations revealed that the locations and strengths of these streaks were generally consistent with those observed at 0.6-degree. This again suggests that these streaks were more or less vertically aligned. Furthermore, Figure 11 shows that (i) streak III was associated with high-speed gap flow across the valley between Lantau Peak and Sunset Peak, (ii) streak I was associated with flow around the western side of Lantau Peak, and (iii) streak II was associated with flow around the eastern side of Lin Fa Shan. However, streak I at low levels (observed at 0.6- and 1.0-degree) was somewhat narrower than the corresponding streak I at 2.4-degree elevation. One explanation for this was that streak I at low levels originated from high-speed gap flow across the valley between Nei Lak Shan and Lantau Peak. However, without additional observational data and diagnostics by numerical models, it is difficult to know the actual trajectories of the airflow within these streaks and the mechanism for the apparent downward transport of momentum from aloft.

\section{CONCLUDING REMARKS}

Vortex shedding and the existence of von Kármán vortex streets in the wake of islands is a well-known phenomenon from cloud patterns with the advent of meteorological satellite. Numerical and field studies of stably stratified flow across islands and isolated obstacles have provided insights to the mechanisms of their formation ${ }^{7,8}$. There are also recent observational and numerical studies of potential vorticity (PV) banners on the lee of the complex terrain of the Alpine region $^{9,10}$. However, we are not aware of observational studies of wake flow of complex terrain with a scale similar to the present case in near-neutral conditions. The TDWR observations presented here are the first data sets which provided highresolution information in both space and time on the structure and evolution of airflow down the wake of such a complex terrain in near-neutral conditions. Ongoing studies are being conducted to better understand the cause and behaviour of these phenomena, and to improve on the operational system for detecting and warning terrain-induced wind shear for the HKIA.

In the present study, we identified reverse flows, vertically-aligned streaks, small-scale vortices and high-speed gap flows induced by the complex terrain of Lantau in strong near-neutral southerly flow associated with tropical cyclones. These phenomena could induce strong shear regions which led to significant low-level wind shear for landing/departing aircraft. There is also evidence of vertical transport of vorticity and momentum. Although rotors with horizontal axis were not observed in low altitudes, the reverse flows on the lee slope of the various peaks coupled with the ambient flow aloft could suggest their existence at higher altitudes. Vertical winds observed by wind profilers installed over Lantau Island could provide further insight ${ }^{4}$. With recent advances of high PRF coherent Doppler LIDAR for remote wind sensing, the Hong Kong Observatory is planning to install such an equipment at the HKIA to supplement the existing facilities for wind shear and turbulence detection. By conducting Range Height Indicator (RHI) scans using the LIDAR, we expect that more information could be gathered on the vertical structure of the wake flows so that the mechanisms for the vertical transport of 
vorticity and momentum could be better understood and the existence/non-existence of rotors might be confirmed. Numerical studies will also be explored to assist in diagnosis of the observed phenomena, in view of the fact that the previous numerical study of turbulence over Lantau in near-neutral conditions only concentrated on southeasterly flow ${ }^{11}$.

\section{ACKNOWLEGMENTS}

We gratefully acknowledge the support of Cathay Pacific Airways Ltd which provided the flight deck data used in this study and the assistance of pilots for filing wind shear reports to the Hong Kong Observatory for the purpose of enhancing flight safety. We would also like to thank the US Federal Aviation Administration for making available the WxShell Software of the Lincoln Laboratory of the Massachusetts Institute of Technology to us for visualizing TDWR data. We also appreciate the work of Miss Olivia Lee in preparing the various figures.

\section{REFERENCES}

1. C.M. Shun and D.B. Johnson, "Implementation of a Terminal Doppler Weather Radar for the new Hong Kong International Airport at Chek Lap Kok", Preprints, Sixth Conf. on Aviation Weather Systems (Dallas, Texas), American Meteorological Society, Boston, pp. 530-534, 1995.

2. D.B. Johnson, R.J. Keeler, C. Kessinger, C.M. Shun, P. Wilson and J.G. Wieler, "Optimization and Testing of a Terminal Doppler Weather Radar for the new Hong Kong International Airport at Chek Lap Kok", Preprints, $28^{\text {th }}$ Conf. on Radar Meteorology (Austin, Texas), American Meteorological Society, Boston, pp. 174-175, 1997.

3. P.P. Neilley, G.B. Foote, T.L. Clark, L.B. Cornman, H. Hsu, T.L. Keller, J. Tuttle and A.R. Rodi, "Observations of Terrain-Induced Turbulent Flow in the Wake of a Mountainous Island", Preprints, $7^{\text {th }}$ Conf. on Mountain Meteorology (Breckenridge, Colorado), American Meteorological Society, Boston, pp. 264-269, 1995.

4. S.Y. Lau and C.M. Shun, "Observation of Terrain-Induced Windshear around Hong Kong International Airport under Stably Stratified Conditions", Preprints, $9^{\text {th }}$ Conference on Mountain Meteorology (Aspen, Colorado), American Meteorological Society, Boston, pp. 93-98, 2000.

5. D. Turnbull, J. McCarthy, J. Evans and D. Zrnic, "The FAA Terminal Doppler Weather Radar (TDWR) Program", Preprints, $3^{\text {rd }}$ International Conference on the Aviation Weather System (Anaheim, California), American Meteorological Society, Boston, 1989.

6. M. Michelson, W.W. Shrader and J.G. Wieler, "Terminal Doppler Weather Radar", Microwave Journal (February issue), pp. 139-148, 1990.

7. R.B. Smith and V. Grubišic, "Aerial Observations of Hawaii's Wake", J. Atmos. Sci., 50, pp. 3728-3750, 1993.

8. R.B. Smith, A.C. Gleason, P.A. Gluhosky and V. Grubiš ic, "The Wake of St. Vincent", J. Atmos. Sci., 54, pp. 606-623, 1997.

9. L.B. Nance, J.-L. Attie, B. Benech, B.R. Colman, J.D. Doyle, C.N. Flamant, V. Grubiš ic, J. Pelon, F.M. Ralph, R. Rotunno, C. Schär, R.B. Smith and R. Steinacker, "Observations of Potential Vorticity Banners during the Mesoscale Alpine Programme", $9^{\text {th }}$ Conference on Mountain Meteorology (Aspen, Colorado), American Meteorological Society, Boston, pp. 43-48, 2000.

10. C. Schär, M. Sprenger, D. Lüthi, R. Benoit and Q. Jiang, "Comparison of In-Situ PV Banner Observations against High-Resolution Numerical Simulations during MAP", $9^{\text {th }}$ Conference on Mountain Meteorology (Aspen, Colorado), American Meteorological Society, Boston, pp. 49-53, 2000.

11. T.L. Clark, T. Keller, J. Coen, P. Neilley, H. Hsu and W.D. Hall, "Terrain-Induced Turbulence over Lantau Island: 7 June 1994 Tropical Storm Russ Case Study", J. Atmos. Sci., 54, pp. 1795-1814, 1997. 


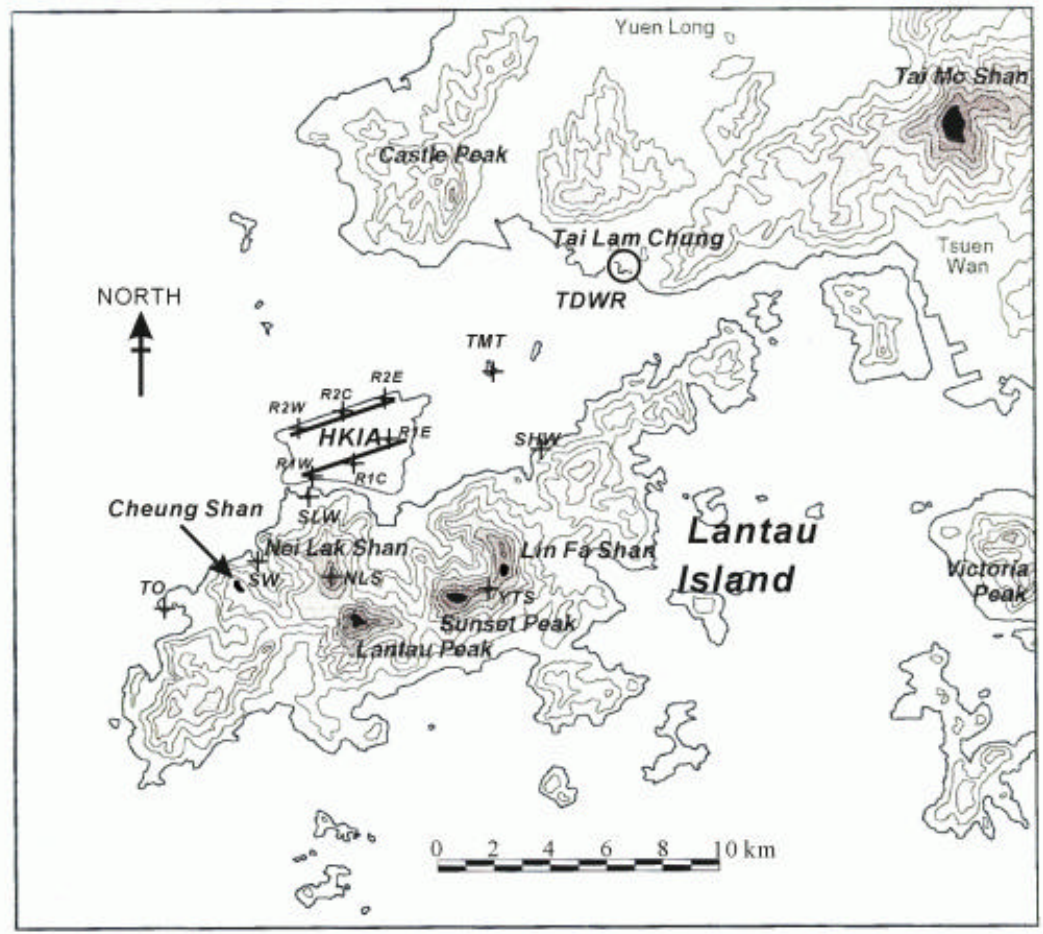

Figure 1. Map of HKIA and its surrounding areas. Terrain contours are given in $100 \mathrm{~m}$ intervals.

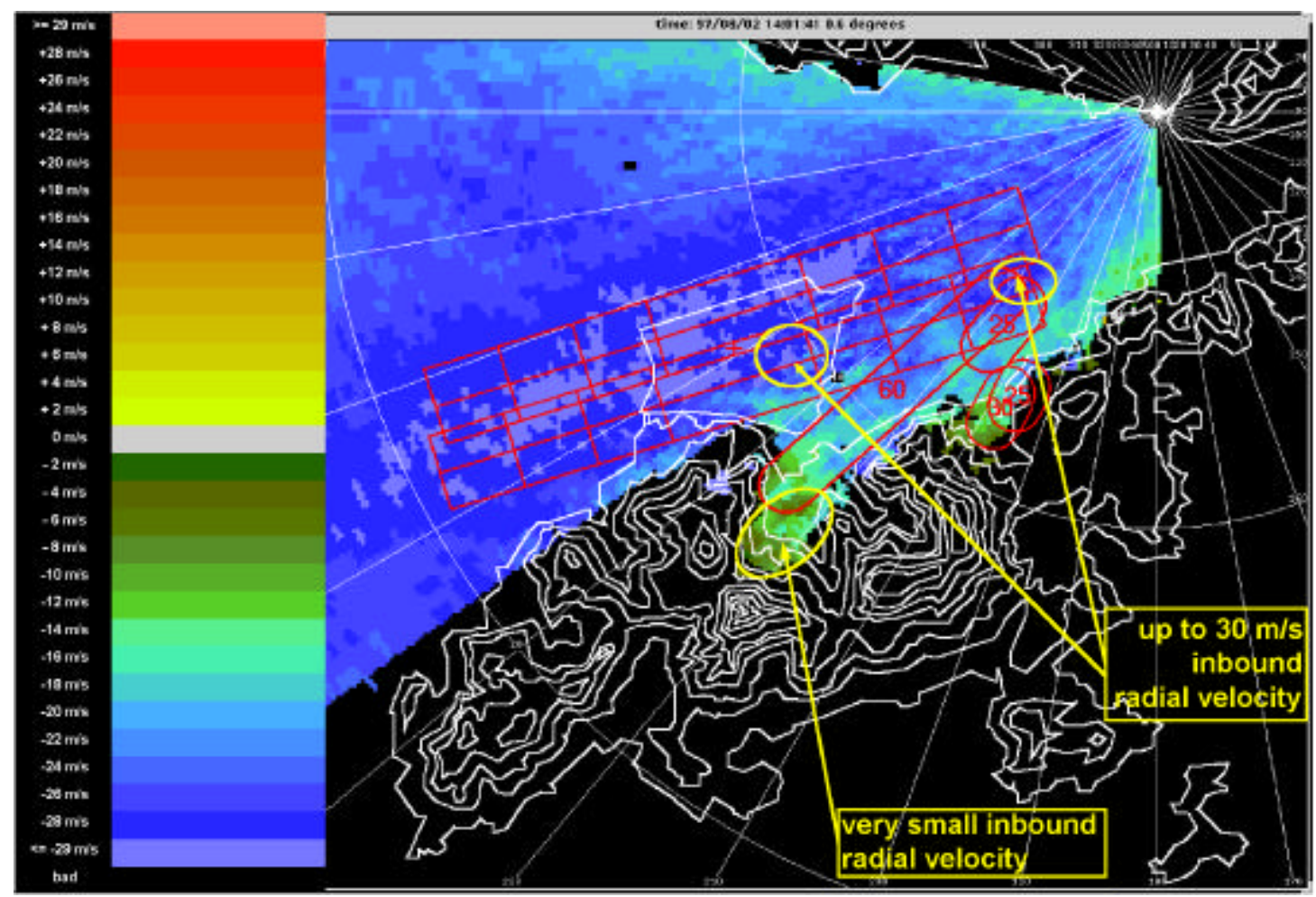

Figure 2. TDWR radial velocity data at 0.6-degree elevation at 14:02 UTC on 2 August 1997. 


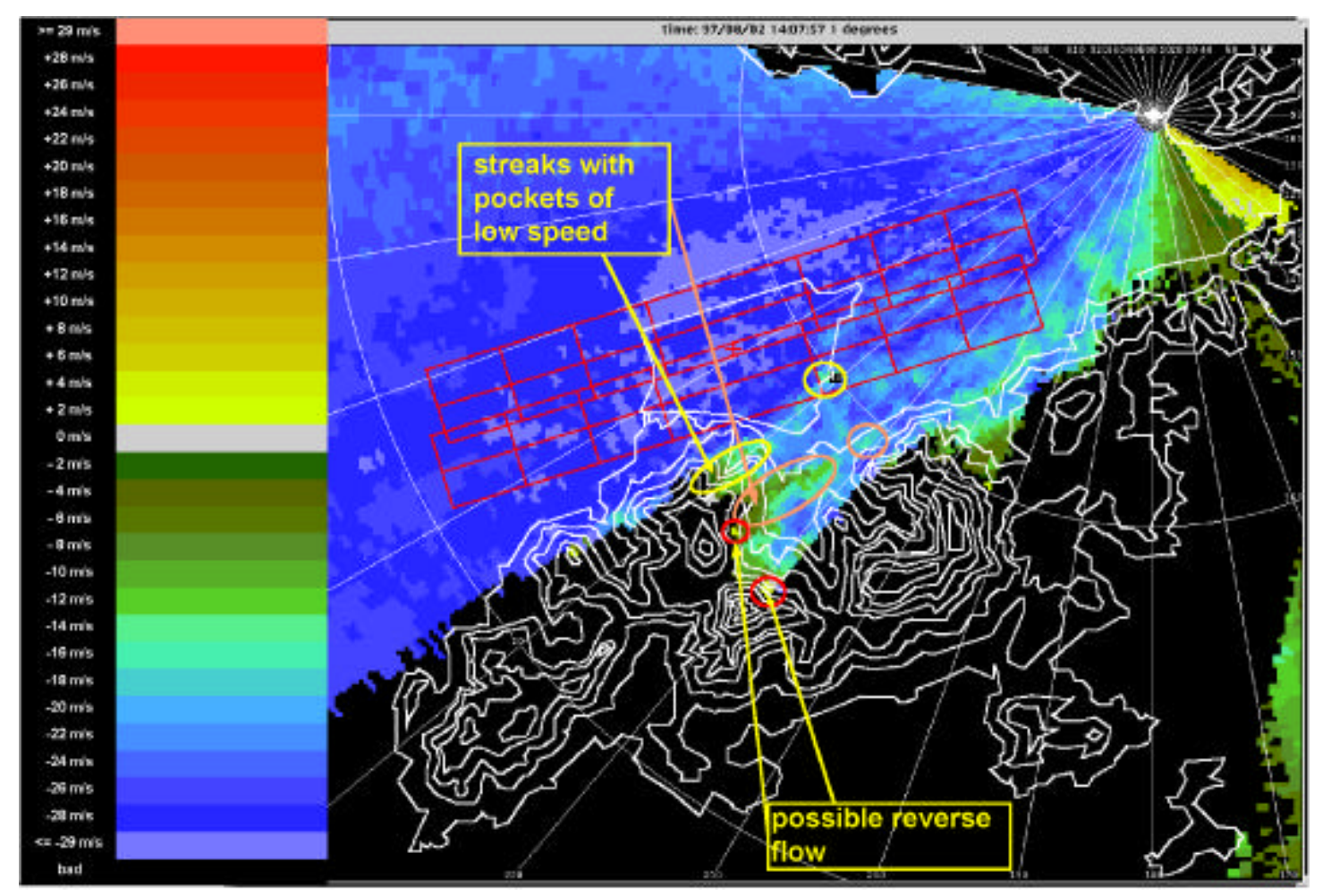

Figure 3. TDWR radial velocity data at 1.0-degree elevation at 14:08 UTC on 2 August 1997.

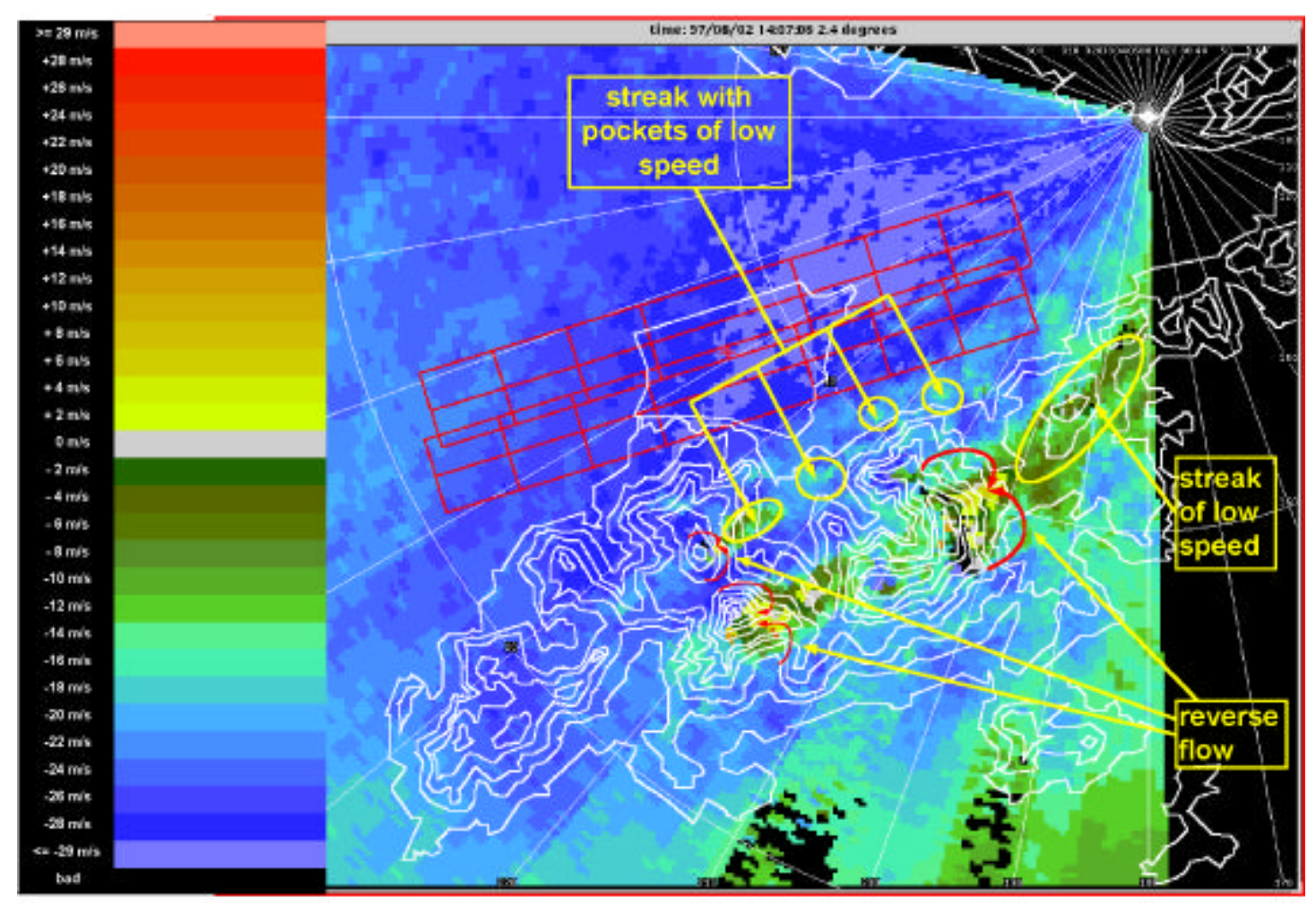

Figure 4. TDWR radial velocity data at 2.4-degree elevation at 14:07 UTC on 2 August 1997. 


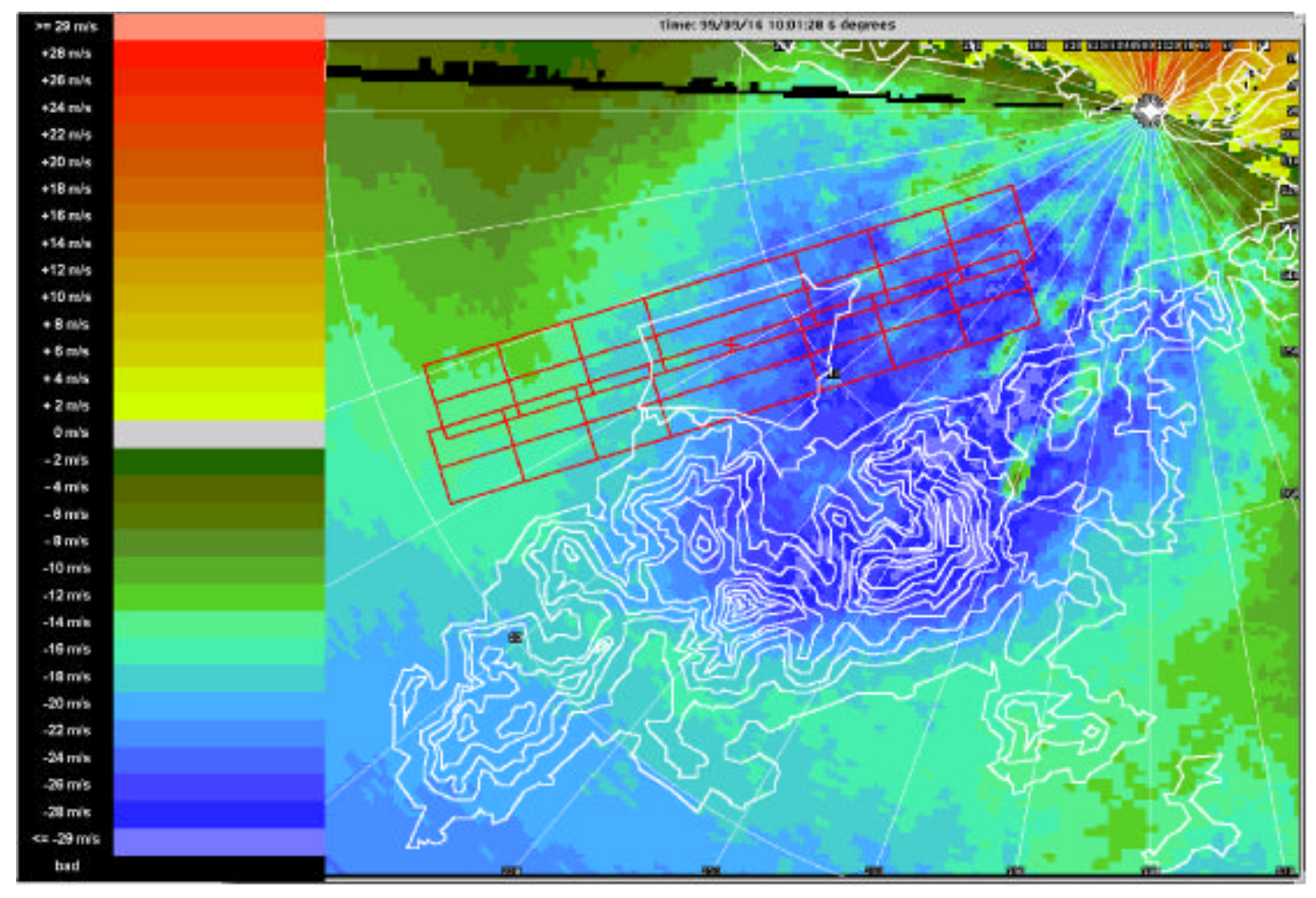

Figure 5. TDWR radial velocity data at 6.0-degree elevation at 10:01 UTC on 16 September 1999.

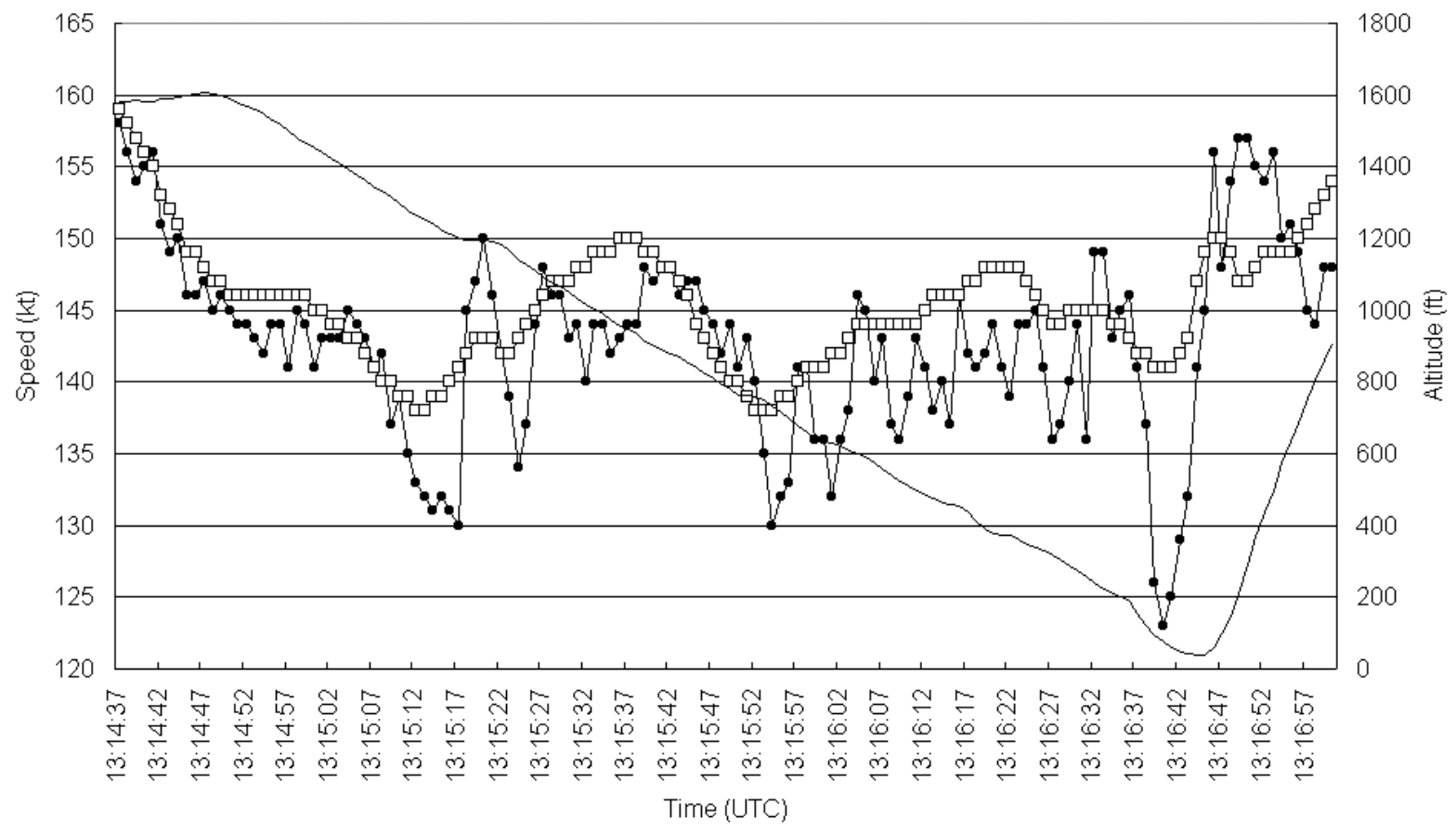

$\rightarrow$ Air Speed $\multimap-$ Ground Speed —-Altitude

Figure 6. On-board flight data at 1-second resolution of an aircraft which went around at 13:16 UTC on 7 June 1999. 


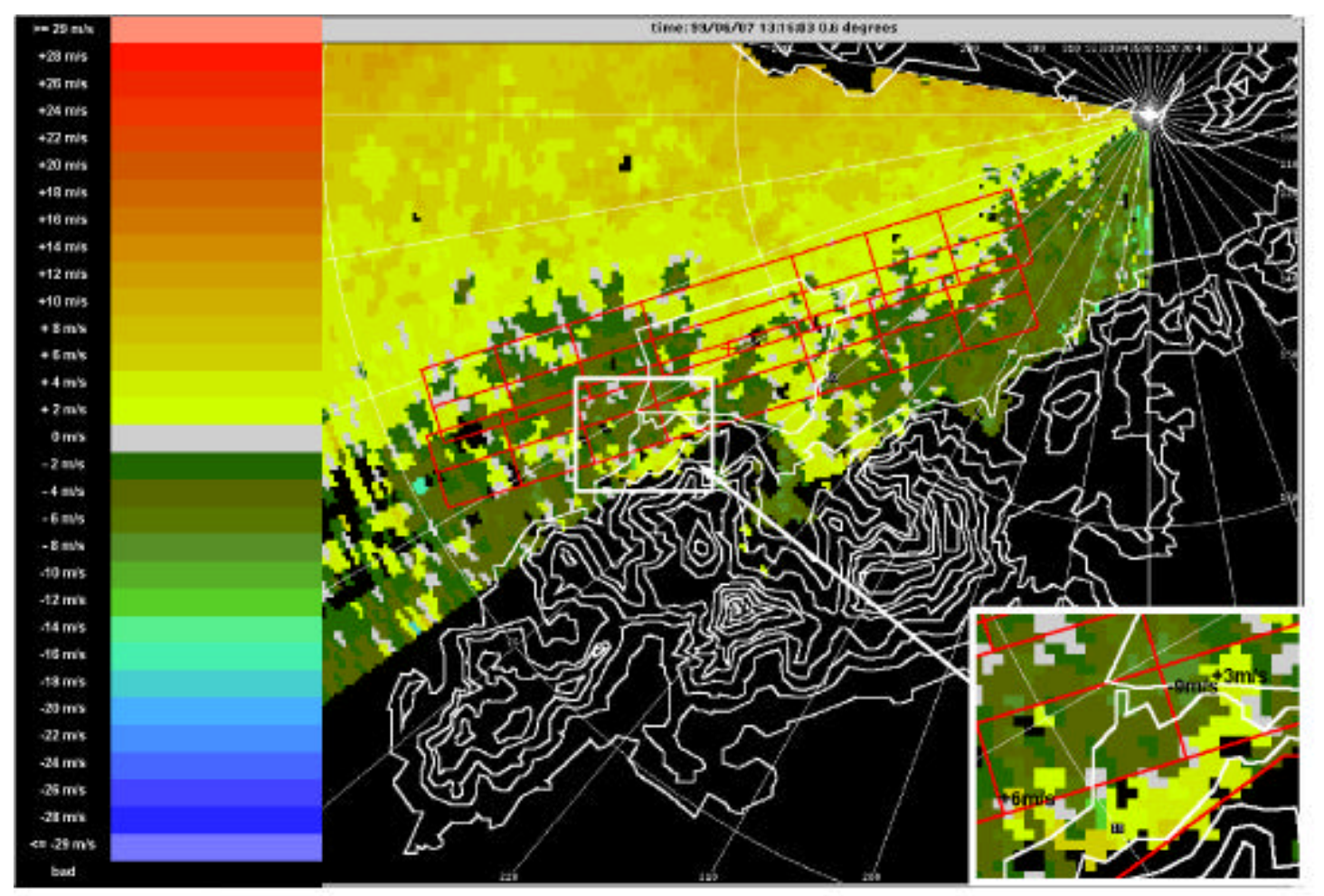

Figure 7. TDWR radial velocity data at 0.6-degree elevation at 13:16:03 UTC on 7 June 1999.

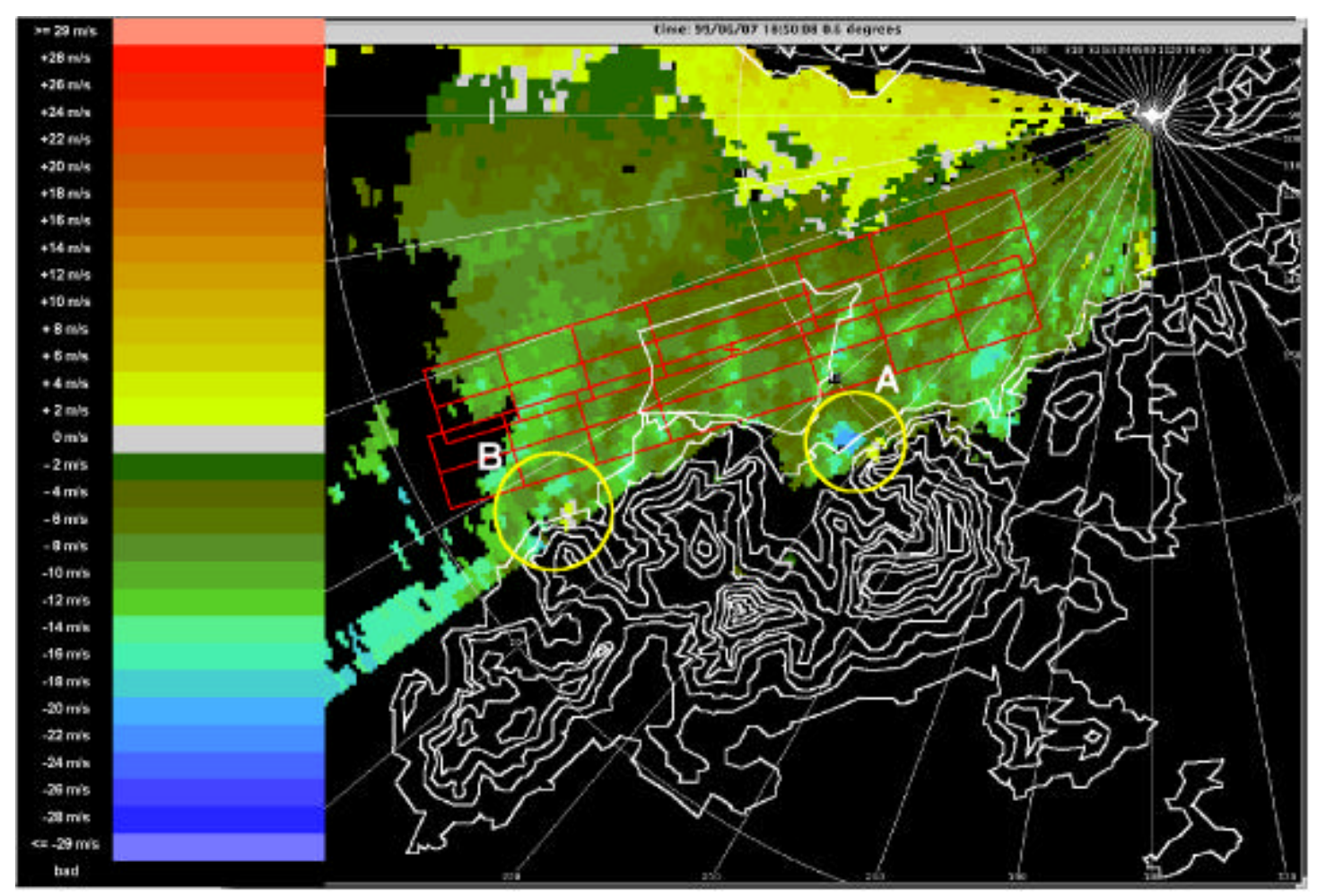

Figure 8. TDWR radial velocity data at 0.6-degree elevation at 18:50 UTC on 7 June 1999. 


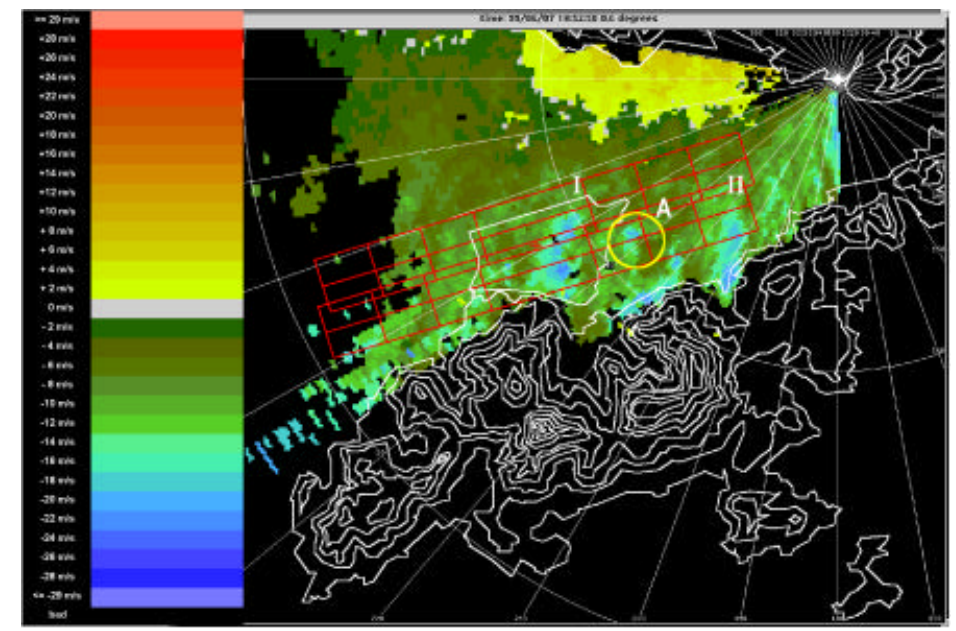

Figure 9. TDWR radial velocity data at 0.6-degree elevation at 18:53 UTC on 7 June 1999.

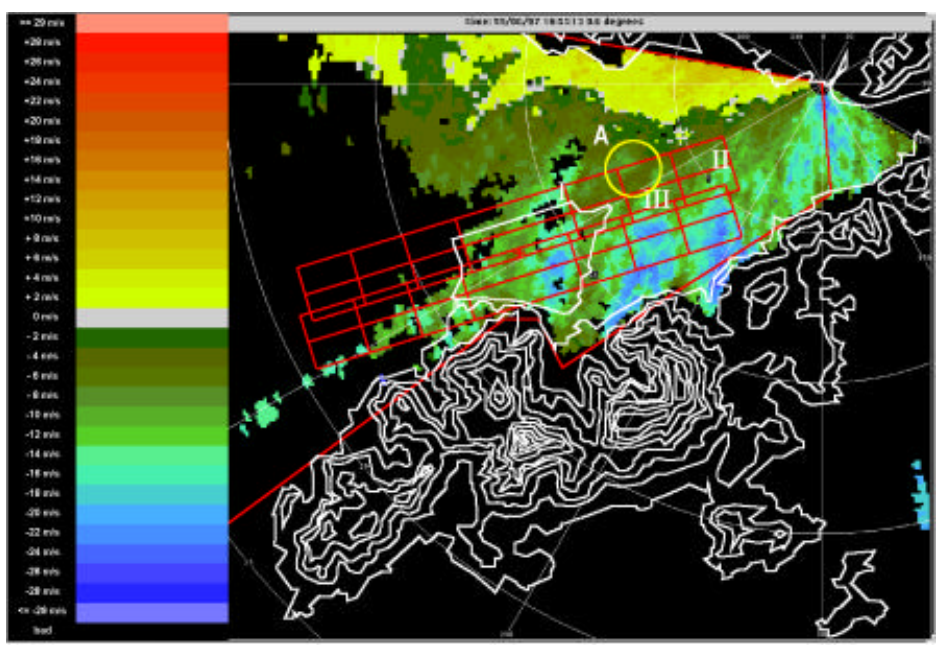

Figure 10. TDWR radial velocity data at 0.6-degree elevation at 18:55 UTC on 7 June 1999.

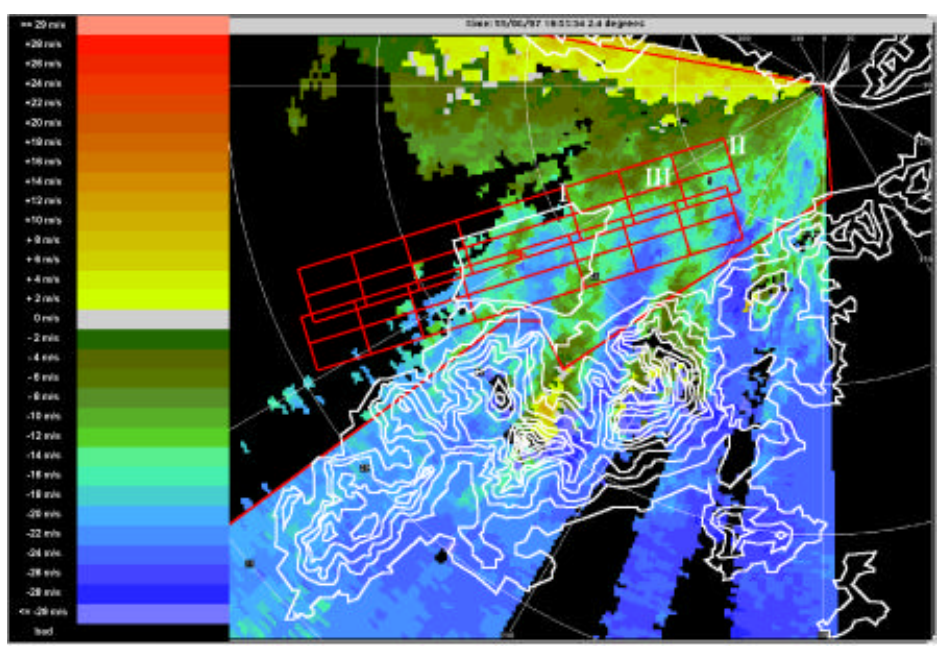

Figure 11. TDWR radial velocity data at 2.4-degree elevation at 18:56 UTC on 7 June 1999. 